\title{
Pilbara rock art: laser scanning, photogrammetry and 3D photographic reconstruction as heritage management tools
}

\author{
Annabelle Davis ${ }^{1 *}\left(\mathbb{D}\right.$, David Belton², Petra Helmholz², Paul Bourke ${ }^{3}$ and Jo McDonald ${ }^{4}$
}

\begin{abstract}
Recording techniques such as laser scanning, photogrammetry and photographic reconstruction are not new to archaeology. However as technology evolves and becomes more readily available such methods are being more regularly employed within a cultural heritage management context, often by people with little experience in using these technologies for heritage applications. For most cultural heritage management practitioners, the awe and lure of technology and the ease with which it can bring archaeology to life can distract from the end game of managing the site on the ground. This paper examines the advantages and disadvantages of laser scanning, photogrammetry and photographic reconstruction in recording, managing and interpreting rock art sites with an emphasis on its practical applications to the field of heritage management. Using a case study from West Angelas in the East Pilbara region of Western Australia, we will examine how these technologies assist in the practical management of heritage sites, and the significant outputs achieved for Aboriginal stakeholder groups in remote access to, and the interpretation of indigenous heritage sites.
\end{abstract}

Keywords: Rock art, Photogrammetry, Laser scanning, Photographic reconstruction, 3D modelling

\section{Background}

In the last two decades, a large number of hardware and software solutions have become available that enable the $3 \mathrm{D}$ reconstruction of heritage features in the form of a 3D coloured point cloud or textured mesh of objects based on laser scanner data and 2D imagery. As a result, 3D reconstruction has become a popular and common tool in the field of archaeology and heritage management [1]. The number of available technologies for the reconstruction and management of cultural heritage continue to increase, but there has been a lack of critical evaluation of the potentials and limitations of these different recording techniques. This paper reports on the outcomes of a high resolution digital recording project at three rock art sites located in the East Pilbara region of Western Australia (WA). Whilst the project's primary aim was to produce a comprehensive, high-resolution, in-perpetuity

\footnotetext{
*Correspondence: annabelle.davis@riotinto.com

${ }^{1}$ Rio Tinto, Communities Division, PO Box A42, Perth, WA 6837, Australia Full list of author information is available at the end of the article
}

record of known rock art, the methods used in the study also provided the opportunity to examine the advantages and disadvantages of these methods to rock art recording, heritage management and community engagement. Whilst the case study is focussed on rock art the methods and results may be transferable to other types of archaeological evidence and cultural heritage management.

This paper provides background information including a review of existing literature and an introduction to the study area. We present the data capturing and processing methods discuss the benefits and disadvantages of each of these methods in detail. After describing the deliverables and products we discuss how the techniques deployed met the research and management goals of the project and make conclusions about the utility of these approaches in heritage management practise.

\section{Technical background}

The technology of deriving 3D measurements based on a set of overlapping images is traditionally referred to as the field of photogrammetry. Photogrammetry uses 
organised and structured recording methods to capture the object of interest. A more recent development, using techniques originating in the domain of machine vision, is the reconstruction of full 3D models from a large collection of overlapping photographs. Compared to photogrammetry-based approaches which produce a more rigorous metric result, photographic 3D reconstruction approaches are usually undertaken in a less structured manner. A statistical analysis of the success of the 3D reconstruction rarely occurs and the reconstruction is considered a success if it "looks right".

In the field of archaeology; software packages such as Arc3D, Photosynth/Bundler, AutoDesk PhotoFly and Photoscan are often used for 3D photographic reconstruction [2-4]. Publications in this field to date have focussed on the intuitiveness of reconstruction software, but the achievable accuracy within these systems is rarely assessed [2]. And yet, geometric accuracy of 3D reconstructions is very important when undertaken in a preservation/reconstruction context [6], to enable measurements to be made reliably and accurately. Westoby et al. [5] captured a 3D point cloud using a laser scanning system and associated images and compared the point density of both sets of point clouds. However, the accuracy of this data was not considered in their review. There are two main methods of assessing the geometric accuracy of $3 \mathrm{D}$ reconstructions currently practiced in archaeology; namely comparison of the reconstructed data to real world measurements and comparison of data capture from multiple sources [4]. In the reconstructed data to real world measurements comparison, the distances of points in the 3D reconstruction result are compared with distances observed in the real world, i.e. directly with the object of interest. When Koutsoudis et al. [4] applied this method they found that the difference of the distances using this technique was small: less than $1 \mathrm{~cm}$. In the second type of comparison test the point cloud created from the dense image reconstruction is compared with the point cloud captured from a terrestrial laser scanning (TLS) system which is used as the reference. This method revealed differences between methods that were much larger, greater than $5 \mathrm{~cm} \mathrm{[4]}$.

A number of publications [7-9] have examined the combination of using laser scanners and images, however these projects only used images to colour the point cloud rather than for $3 \mathrm{D}$ reconstruction. Compared to the use of images, the use of laser scanners requires more equipment and software. Specific training in the operation of the scanners and a more careful planning of the capturing process is required, due to the complexity of the set up time and safety issues related to using a laser. A number of researchers have used MeshLab software to align the different scans taken from the different parts of the object of interest [7-9]. As with the photographic $3 \mathrm{D}$ reconstruction software solutions, MeshLab does not provide any statistical values to indicate the precision or accuracy of the alignment of the point clouds. Furthermore, accuracy checks are often not performed in this type of work.

This project utilised traditional rock art recording techniques and three additional data collection methods: terrestrial laser scanning (TLS), photogrammetry and photographic 3D reconstruction. All solutions create dense 3D point clouds, with 3D reconstruction going one step further to create textured 3D meshes.

\section{Terrestrial laser scanning}

Terrestrial laser scanners (TLS) capture 3D information directly, and are used for a variety of applications [10]. TLS measures distances through the use of a laser ranging system (based on time of flight using either pulsed or phase-based measurements), and combines them with the angular measurement to produce three dimensional information in the form of points, generating a point cloud over a field of view. The type of scanning technology has an impact on the accuracy, maximum range and speed of acquisition. Traditionally, pulse systems operate over greater ranges, whereas phased-based systems have increased accuracy and capture speeds.

Whilst the scanner can only capture objects and structures in direct line of site from the laser position, scans from multiple locations can increase the coverage by being combined through a registration process. Laser scanning also captures returned intensity information, which is a function of the scanner geometry to the surface and the surface properties such as reflectance and texture in relation to the laser wave-length. Other properties can be mapped onto the points such as colour (red/ green/blue), thermal, and hyper spectral data, either through an on board imaging systems or the registration of images from external sources. The generated point cloud can be processed further to produce meshed surfaces, models and/or drawings.

The costs for surveying grade scanners can range over an order of magnitude, from tens of thousands to hundreds of thousands of dollars. Additional costs also include tripods, targets and data processing software, amounting to tens of thousands of dollars.

\section{Photogrammetry}

Photogrammetry enables precise 3D measurements using images [11]. Careful planning of the data capture is required depending on the required level of detail. To be able to extract precise measurements, the geometry in which the images are captured, the quality of the cameras and lenses used and the in situ calibration of the camera 
are all important. The camera requires sufficient light to illuminate the scene (this can be natural light or spot lights). Homogenous illumination of the scene with no shadow or reduced shadow effects is desirable.

From the overlapping images of the object of interest, the position of the camera can be determined. Using trigonometry the position of any object visible in at least three images can be calculated in $3 \mathrm{D}$ space. These measurements can be done for discrete points or alternatively a dense $3 \mathrm{D}$ point cloud can be created. The metrics related to these 3D measurements can then be added using global control points or a scale bar. The wavelength represented in the 3D model is defined by the wavelength sensitivity of the camera used [12]. The generated point cloud is then usually processed further to produce a meshed surface.

Photogrammetry costs include the cost of a medium to high range camera including a high grade lens, typically under AU $\$ 10,000$, as well as suitable software which can range from open source to AU\$25,000 with high cost software solutions normally offering better statistical measurements about the quality of the photogrammetric processing.

\section{Photographic reconstruction}

Whilst the techniques discussed above have a long and rich history, 3D reconstruction is a relatively new technology which is still seeing a steady improvement in the quality of the algorithms and subsequent outcomes.

As with the photogrammetric approach, photographic reconstruction employs images and an estimated position of the cameras to derive a point cloud. It differs in that the algorithms employed benefit from a larger number of photographs and rather than stop at a point cloud, the goal is to create $3 \mathrm{D}$ meshes with a high texture quality. A reconstructed model has two components: the texture and the underlying geometry. The high quality textures can often lead to a perceived geometric resolution that may not be actually represented in the reconstructed geometry. An important benefit of this approach is that every photograph is ideally captured from a different position: subject to access limitations this means that shadow zones can be kept to a minimum. Both photogrammetry and 3D reconstruction don't suffer from the parallax effects that arise from colouring point clouds from laser scanners where the camera is not coincident with the laser source.

It should be noted that such a reconstructed model generally is of arbitrary scale and orientation. Additional real world reference points or distances can be used to constrain the reconstruction, or applied to the model as a post-production stage in much the same way as for photogrammetry.
The costs for the camera system are similar to the camera costs for photogrammetry, at most $\$ 10,000$ for a camera, tripod and quality prime lens.

\section{Case study}

The rock art sites that were the subject of this study are located at Rio Tinto's West Angelas mine site in the East Pilbara, within the Yinhawangka native title claim area (Fig. 1).

Whilst currently being managed in situ the sites are at risk of indirect impacts from mining activity. One site, in particular is located within immediate proximity of an existing operational mine pit. Consultation with the Yinhawangka Traditional Owners determined that a comprehensive set of baseline information in relation to the sites and their key features was required to assist in their ongoing management. The proximity of these sites to mining activity meant that high resolution measurable data such as that which can be achieved from laser scanning will assist in monitoring site condition, and any potential changes in this, over time. Given the cultural significance of the sites to the Yinhawangka people, visualisation and interpretation of the sites was also considered an important community output. In order to meet these combined requirements, traditional archaeological recording, laser scanning and 3D photographic reconstruction methods were undertaken to provide a detailed baseline record of the rock art.

The three sites subject to the study are listed (Table 1).

\section{Data capturing and processing \\ Field preparation}

To carry out the data capture, an initial survey was conducted by the Rio Tinto's West Angelas mine surveyors. This was done to help combine the different data into a single coordinate system and to position this absolutely in reference to a global co-ordinate datum.

The first step was to introduce global coordinates to the site. For the purpose of this survey, relative accuracy was of more concern than absolute accuracy, so Global Navigation Satellite Systems (GNSS) observations were chosen. There are several methods for GNSS that could have been employed (Table 2), with different accuracies, however Real-time Kinematic (RTK) was used since the raw observations could be corrected by the observations from a base station operated on site to within a few centimetres, and the coordinates could be calculated within seconds. If this base station was not available, then other methods such as Differential GPS (DGPS), and AUSPOS could be used to improve the raw data accuracy [14]. These control points could then be used to calculate the rotation and translation of the local system to the global system. 


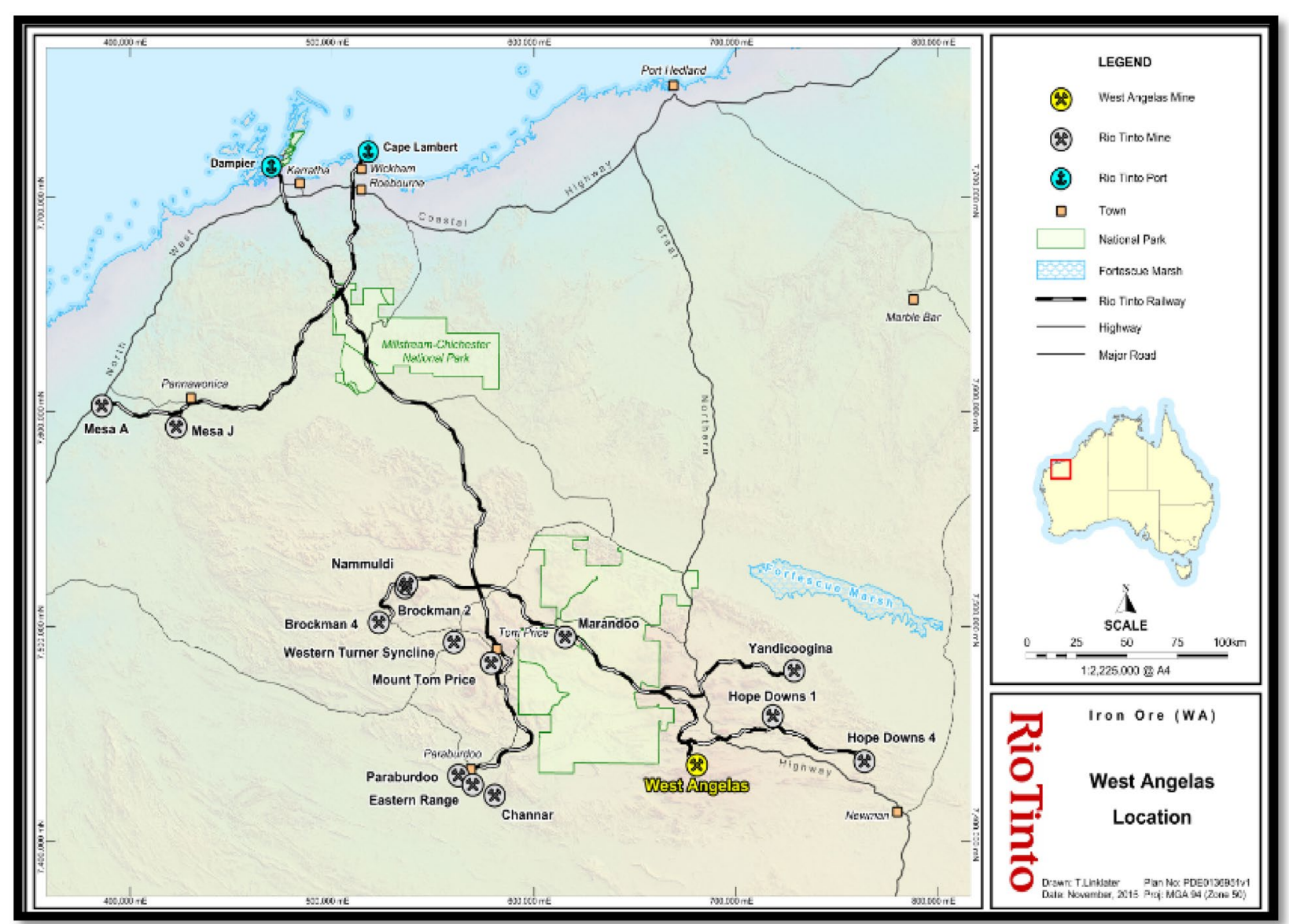

Fig. 1 Study area location

For the local system, targets were placed around the area that both the imagery and laser scanners could identify and capture. The co-ordinates for these targets with respect to the control points were calculated using the TLS in a similar manner to how a traditional survey would be carried out using a total station. This created a network such that the positions of these targets could be used to calculate the rotation and translation between the different setups and the different methods of capture in order to put them in a common reference system, and for the photogrammetry to introduce scale information into the models.

\section{Rock art recording}

After locating the sites, the archaeologists conducted a fine-grained inspection of the sites' walls and other suitable surfaces in order to identify any previously unidentified motifs and to determine the scope of the required recording. This inspection revealed the presence of previously undocumented pigment and engraved motifs at all sites. All motifs/features were recorded in detail during the current exercise.

All field data was recorded onto iPads loaded with a FilemakePro13 database developed by CRAR $+M$ for rock art in the Pilbara [15]. A Canon EOS 5D DSLR camera with a 35-105 mm lens was used for digital photography, as was a Canon G12 with the D-Stretch plugin loaded on its card [16]. D-Stretch image enhancement software on the camera in the field allows for full identification of faint motifs on panels, and ensures complete photographic capture of faint motifs. Line drawings (sketches) of pigment art were done where required. A Dinolite digital microscope/camera was used to observe superimposition detail (where applicable) and pigment condition (see Fig. 2).

In addition to the archaeological recording, a detailed separate ethnographic consultation was also undertaken in relation to the cultural significance of the sites [17]. Senior and younger Yinhawangka representatives participated in all aspects of the project. 
Table 1 Site descriptions. Adapted from [13]

\begin{tabular}{|c|c|c|}
\hline Site & Situational context & Rock art details \\
\hline $\begin{array}{l}\text { DE-SH2 (DAA } \\
\text { 20444) }\end{array}$ & $\begin{array}{l}\text { Extensive rockshelter with an easterly aspect } \\
\text { at the base of a low escarpment overlook- } \\
\text { ing a gentle colluvial slope }\end{array}$ & $\begin{array}{l}\text { Four separate panels at the shelter: two in the main chamber, one on the exterior wall } \\
\text { and the fourth in the second chamber. The art in the main chamber consists of a } \\
\text { red hand print on the wall and a roof fall block on the floor with pecked pits and an } \\
\text { engraved bird track. On an exterior wall between the two chambers is a faint yellow } \\
\text { painted geometric motif (tally marks) and in the second chamber is another painted } \\
\text { set of yellow tally marks }\end{array}$ \\
\hline DF-SH1 & $\begin{array}{l}\text { Large shelter located above a small creek } \\
\text { line }\end{array}$ & $\begin{array}{l}\text { Five separate panels including pigment art and pecked pits. Engraved (pecked) pits } \\
\text { are located on a large piece of roof fall. Pigment art consists of a large white geo- } \\
\text { metric motif at the front of the shelter, two other panel containing geometric motifs } \\
\text { in white and red pigment }\end{array}$ \\
\hline $\mathrm{RR} 21$ & $\begin{array}{l}\text { Site complex in an open gorge context with } \\
\text { four vertical faces of pigment art and } 2 \\
\text { rockshelters containing art and/or ochre }\end{array}$ & $\begin{array}{l}\text { Yellow and quite painted motifs (126) predominantly anthropomorphic figures: linear } \\
\text { (stick) figures, solid figures, and groups of anthropomorphic figures. Geometric } \\
\text { motifs include linear geometrics and parallel lines }\end{array}$ \\
\hline
\end{tabular}

Table 2 Global positioning methods [14]

\begin{tabular}{lll}
\hline Method & Position accuracy & Time \\
\hline Raw data & \pm 4 to $10 \mathrm{~m}$ & $<\mathrm{min}$ \\
RTK GNSS & \pm 20 to $50 \mathrm{~mm}$ & $<\mathrm{min}$ \\
DGNSS & $\pm 1 \mathrm{~m}$ & $<\mathrm{min}$ \\
Static baseline & $\pm 10 \mathrm{~mm}$ or better & $15-20 \mathrm{~min}$ \\
Auspos & \pm 0.1 to $0.01 \mathrm{~mm}$ & $4 \mathrm{~h}$ minimum $(8-24 \mathrm{~h})$ \\
\hline
\end{tabular}

\section{Terrestrial laser scanning}

The sites were captured using two different laser scanning systems, the Leica C10 and the Trimble TX5. These two systems were chosen for their different properties (see Table 3). The Leica C10 is a pulse based system using a green laser. This is typically used for medium range survey applications. A larger spot size and slower capture rate means that it lacks resolution to capture fine detail, but its longer range enables it to capture larger scenes with good positional accuracies. The Trimble TX5 is a phased based system using a near-infrared laser. It is more often used for shorter range scene captures, with faster sampling and higher point accuracies allowing it to capture finer resolutions (although the detail is still limited by the spot size). Both systems also have integrated imaging systems to colour the point cloud directly.

Two of the three sites (DF-SH1 and DE-SH2) were captured using both scanners, with data captured from the surrounding area and inside the rock shelters. Using the geo-referenced controls described above, the final coordinates had an accuracy of 2-5 cm depending on conditions (slope and vegetation).

To make use of the different capabilities of the two scanners, the capture was separated into different stages (Table 4). The C10 was used primarily to capture the large area surrounding rock shelters to take advantage of its longer range. Several setups were taken to scan the gully and surrounding areas, as well as scanning the inside of the rock shelter. This allowed the landscape to be captured to provide context to the rock shelter and the art it contained. On average, each scan from the C10 required between $12 \mathrm{~min}(1 \mathrm{~cm}$ resolution at a range of $10 \mathrm{~m})$ to $40 \mathrm{~min}(5 \mathrm{~mm}$ resolution at a range of $20 \mathrm{~m})$. Several scans from the TX5 were then captured inside the rock shelter. Its higher resolution and accuracy allowed the capture of more detail inside the shelter. Each scan of the TX5 took an average of $7 \mathrm{~min}$ with a resolution of $2 \mathrm{~mm}$ at a distance of $10 \mathrm{~m}$.

The different scans were registered into the common coordinate system using the Leica Cyclone software. This made use of the captured targets to calculate the transformation parameters between the scans, and the geo-referenced control points. A cloud to cloud registration, based on the Iterative Closest Point (ICP) method [20] was also used for scans lacking targets and to improve the final registration by minimising the separation between overlapping point clouds. The final result was a point cloud with absolute positioning of $\pm 2 \mathrm{~cm}$, a relative positioning accuracy over the area of $\pm 5 \mathrm{~mm}$, and a point accuracy ranging from \pm 6 to $\pm 1 \mathrm{~mm}$. Point spacing ranges from 2 to $5 \mathrm{~cm}$ around the surrounding areas of the rock shelters to within 1-10 mm inside the shelter.

Due to the remoteness of site RR21 this location was captured only with the TX5. Capture here involved multiple setups, and the point clouds were registered based on cloud to cloud registration. Cloud to cloud registration is not as quick as the processes described using targets, and in this instance due to the lack of defined features and heavy vegetation, the process was difficult. Removal of vegetation would have assisted this to a degree, however the practicalities of vegetation removal needs to be considered, particularly in terms of other site management considerations. 


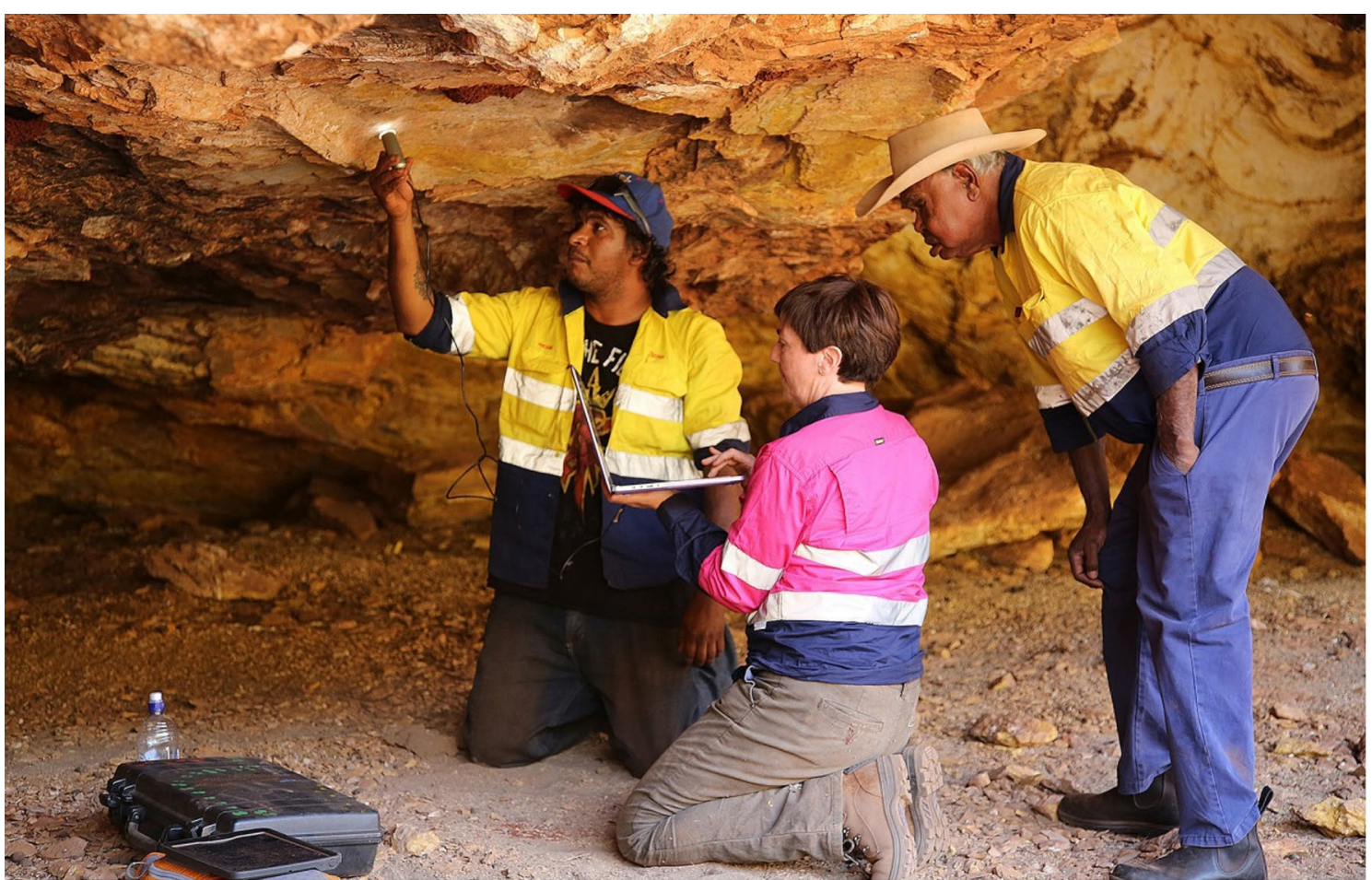

Fig. 2 Right to left David Cox, Jo McDonald and Clayton Parker using the DinoLight for microscopic analysis of pigment art

Table 3 Summary of the two TLS systems used in this project $[18,19]$

\begin{tabular}{lll}
\hline & Leica C10 & Trimble TX5 \\
\hline Point spot size $(\mathrm{mm})$ & 4.5 & 3 \\
Point accuracy $(\mathrm{mm})$ & 6 & 2 \\
Model accuracy $(\mathrm{mm})$ & 2 & 0.6 \\
Maximum distance & $120 \mathrm{~m}($ up to $350 \mathrm{~m})$ & $50 \mathrm{~m}$ (up to $120 \mathrm{~m})$ \\
Scan rate (pts/second) & 50,000 & 976,000 \\
Scan type & Pulsed & Phased based \\
Wave length & Green $(532 \mathrm{~nm})$ & Near infrared $(905 \mathrm{~nm})$ \\
& &
\end{tabular}

\section{Photogrammetry}

A Canon EOS 6D DLSR was used to capture the data. This camera is a full frame camera that can take images of up to 20.2 MP resolutions with dimensions of $5472 \times 3648$ pixels. A Canon EF24-70 mm lens was used in place of the stock lens. The camera was handheld with a scale bar mounted on a tripod and placed in the field of view of at least three images (Fig. 3). Photography involved taking images for all sites (Table 5; Fig. 4) keeping the focal length for each of those shots constant.

A scale bar is a calibrated pole built out of a material with little thermal expansion where the distance between two markers (yellow arrows in Fig. 3) is known. The
Table 4 Number of points in the TLS point clouds

\begin{tabular}{|c|c|c|c|}
\hline Site & DE-SH2 & DF-SH1 & RR21 \\
\hline \multicolumn{4}{|c|}{ Number of TLS setups } \\
\hline Faro & 4 & 5 & 9 \\
\hline Leica & 5 & 5 & NA \\
\hline \multicolumn{4}{|l|}{ Number of points } \\
\hline Faro & $1677,473,687$ & $1721,479,841$ & $3587,370,908$ \\
\hline Leica & $19,869,392$ & $87,637,839$ & NA \\
\hline \multicolumn{4}{|c|}{ Average resolution } \\
\hline Faro (mm) & 1 & 2 & 4 \\
\hline $\begin{array}{l}\text { Leica ( } 5 \mathrm{~mm} \text { in } \\
\text { shelter) }\end{array}$ & $15 \mathrm{~mm}$ & $15 \mathrm{~mm}$ & NA \\
\hline \multicolumn{4}{|l|}{ Area covered } \\
\hline Faro & $45 m \times 46 m$ & $23 \mathrm{~m} \times 25 \mathrm{~m}$ & NA \\
\hline Leica & $105 \mathrm{~m} \times 110 \mathrm{~m}$ & $125 \mathrm{~m} \times 128 \mathrm{~m}$ & $130 \mathrm{~m} \times 52 \mathrm{~m}$ \\
\hline
\end{tabular}

scale bar can be used to scale the measurements and the derived point cloud into the metric system (in metres). It is possible to transform this local system into a global system using the coordinates of the laser scanning control points which are also visible in the images (blue arrows in Fig. 3).

The image datasets were processed using the software $i$ WitnessPro [21] for the photogrammetric process and 
SURE [22] as dense image matching software. Alternative available software solutions are Agisoft's PhotoScanPro [23] and VisualSfM [24] which work in a similar manner to $i$ WitnessPro. In this process, points of interest are identified in each image of the dataset [25]. These points

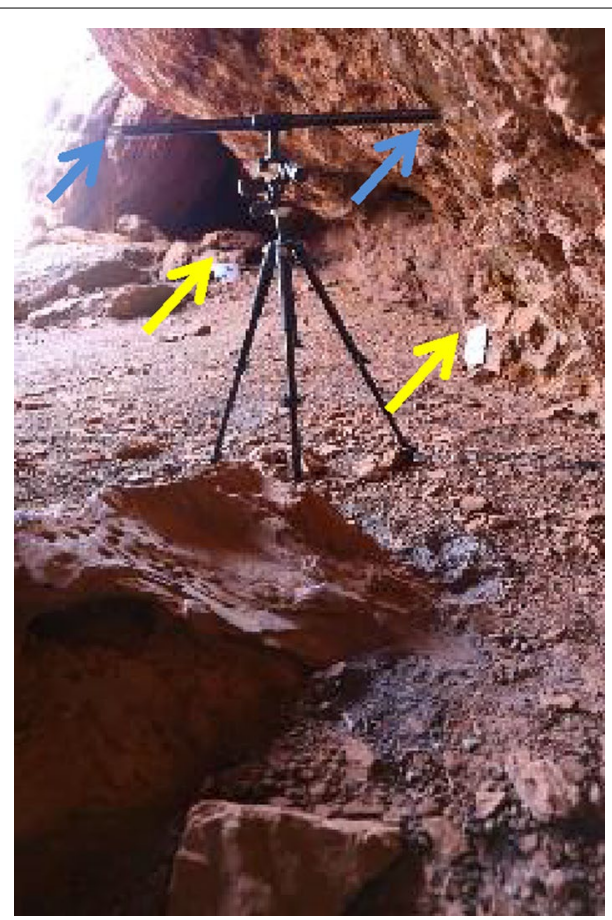

Fig. 3 Scale bar with two markers (yellow arrows) and laser scan targets (blue arrows) placed in the field of view of the image are then matched and corresponding points in the different images are detected. This can be a time consuming process, where the computational complexity (processing time) is in the sum of $O\left(n^{2}\right)$, an exponential expression of time where $n$ is the number of images to be matched. $i$ WitnessPro offers the user the opportunity to match points manually between images whereas VisualSfM (using SIFT) and PhotoScanPro (using an personalised algorithm similar to SIFT) only allows automatic matching. Based on the common points the images are oriented relative to each other applying a bundle adjustment. Compared to VisualSfM, iWitnessPro and PhotoScanPro allow the measurement of the scale bar or control points which are also included in the adjustment. If control points are included in the adjustment an absolute orientation of the images can be done leading to a geo-referenced point cloud in the following step. In order to scale the point cloud in VisualSfM purely an affine transformation in an extra processing step is performed. The bundle adjustment also allows a self-calibration of the camera. This is similar in all three software solutions. However, after the adjustment not only the camera parameters are known, also a sparse point cloud is created (Fig. 4).

As mentioned above, generally the 3D co-ordinates constructed from bundle adjustment are in the form of a sparse point cloud, where the number of points is at most in the region of several hundred thousand. Most modern packages allow for the construction of dense point clouds, either from the sparse point cloud and orientation (such as VisualSFM and PhotoScanPro), or using only the orientation parameters (iWitnessPro in combination with SURE). Unlike their sparse counterparts

Table 5 Number of points in the image based point clouds

\begin{tabular}{llll}
\hline Site & Element & Number of images & $\begin{array}{c}\text { Number of points } \\
\text { in the 3D point cloud }\end{array}$ \\
\hline DE-SH2 & Engravings (cupule) & 28 & Not assessed \\
& Pigment rock art 1 (ceiling, main shelter) & Not recorded & Not assessed \\
& Pigment rock art 2 (outside) & 14 & Not assessed \\
& Pigment rock art 3 (2nd shelter) & 22 & Not assessed \\
DF-SH1 & Engravings (cupule) & 14 & Not assessed \\
& Rock (worktop) & 19 & Not assessed \\
& Pigment rock art 1 (entrance area) & 12 & Not assessed \\
RR21 & Pigment rock art 2 (inside the shelter) & 20 & $70,680,764$ \\
& Site 1 (panel B/C) & 18 & $72,880,169$ \\
& Site 2 (panel D/E/F) & 20 & $84,213,262$ \\
& Site 3 (panel E/F/G) & 19 & $38,719,683$ \\
& Site 4 (panel G/H) & 10 & $60,136,355$ \\
& Site 5 (panel B-H) & 7 & $63,136,874$ \\
& Site 6 (opposite site) & 24 & 17 \\
& Site 7 (opposite site) & 12 & \\
& Site 8 (opposite site) & & \\
\hline
\end{tabular}




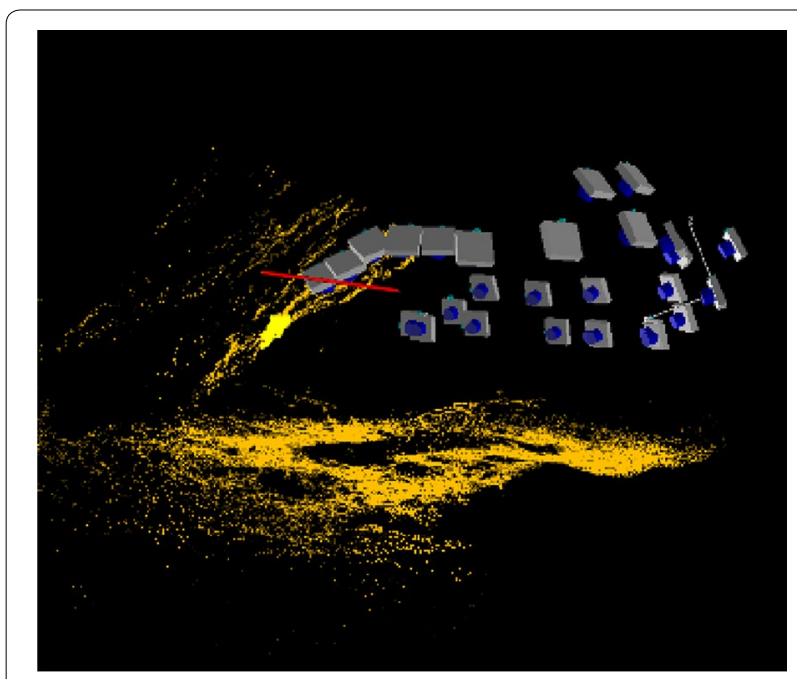

Fig. 4 Sparse point cloud with camera locations and the position of the scale bar (in red)

the dense point clouds can have over a hundred million points. The most common method for dense point cloud matching is called Semi-Global Matching (SGM) [26]. VisualSFM uses a package known as CMVS/PMVS2 [27] to generate dense point clouds, whilst PhotoScanPro's technique is unknown, but based on pair-wise depth map computation [28].

\section{Photographic reconstruction}

The photographic reconstruction was performed using a different camera compared to the previous section, namely a Canon 5D Mark III DSLR camera. The main reason for using two cameras was to speed up the data capturing process as both datasets could be captured at the same time. Both cameras are suitable for both photogrammetry as well as photographic reconstruction. For the reconstruction of the entire rock shelters a $28 \mathrm{~mm}$ prime Sigma lens was employed in order to limit the number of photographs needed for such a large structure. Depending on the shelter, a total of between 200 and 400 photographs were taken. The time required depended on the shelter in question but typically between 15 and $30 \mathrm{~min}$. Other smaller items were photographed with a Sigma $50 \mathrm{~mm}$ prime lens. Between 30 and 50 photographs were taken of each of these more isolated objects.
The 3D reconstructions were created using PhotoScan as well as an in-house pipeline based upon source components (Bundler for feature point and camera pose estimation PMVS2 for dense cloud and mesh creation) [29].

Processing times for the 3D reconstruction vary depending on the number and resolution of the images. It also varies greatly depending on various numerical parameters for each stage of the reconstruction process. Similarly, all stages of the reconstruction pipeline are well suited to parallel processing and take advantage of multiple cores both on the CPU and GPU. All processing for this project was conducted on an MacBookPro $(2.3 \mathrm{GHz}$ Intel Core i7, 16 GB Ram). First pass versions were performed in the field so that the photographic capture could audited, and if necessary, be revisited if for some reason a reconstruction failed or had insufficient coverage or detail. Higher quality reconstructions were performed on the same hardware and took in the order of a few hours each, up to $10 \mathrm{~h}$ for the largest photographic sets of the rock shelters.

\section{Benefits and disadvantages}

In order to determine which technique provides the best fit for the work, a basic understanding of the benefits and disadvantages of each of the methods is required. Table 6 below provides a basic summary of the time, logistical considerations and approximate costs involved with each method. As these factors can be highly variable depending on the nature of the work a low to high scale has been used to differentiate between techniques. These are also further explored below.

\section{Terrestrial laser scanning}

One of the main advantages of using TLS is that it is capable of capturing large areas of survey-quality (accuracy and positional certainty) data. It has a consistent accuracy through the scene and the data is already in 3D format without the requirement for further processing. While the scanner can similarly capture large areas, the need for multiple setups and a higher resolution can result in a much longer field capture time than other methods. The time for each setup depends on the speed of the scanner and the resolution of the point cloud capture, but can range from 12 to $40 \mathrm{~min}$ in the case of the $\mathrm{C} 10$ on this project (from $10 \mathrm{~mm}$ down to $2 \mathrm{~mm}$

Table 6 Comparative times and costs associated with data capture methods

\begin{tabular}{lllll}
\hline Method & Field time & Complexity of logistics & Post-processing time & Costs \\
\hline Terrestrial laser scanning & Low & Medium to high & Medium to high & Medium to high \\
Photogrammetry & Low & Medium & Medium & Medium \\
Photographic reconstruction & Low & Low & Medium & Low \\
\hline
\end{tabular}


resolution and captured over a distance of $10 \mathrm{~m}$ ). This is offset by the fact that the data capture directly in 3D, and requires little extra processing to combine multiple setups into a single point cloud.

The system utilises an active sensor, so it is completely independent from the requirement of an external light source (including the sun and any artificial light source). Furthermore, the wavelength in which the laser operates can be outside of visible spectrum in contrast to a large number of passive sensors such as a standard consumer camera. Often scanners not only capture 3D readings of their environment, but also to information on the reflected intensity of the returned signal to classify surface features based on how strongly the surface reflects the wavelength being used. The reflected intensity information is used in other fields of research such as remote sensing to classify vegetation data [30,31]), and has the potential to reveal structure not visible to the human eye. Some applications of this method to rock art have been attempted but are largely untested [32]. It should be noted that the reverse can also be true; surfaces that may be visually different may not appear different because they may reflect the wavelength of the scanner in an identical manner. Similarly surfaces that reflect energy in parts of the visible spectrum may not reflect energy in the scanner wavelength.

Because the TLS it is an active sensor and takes discrete measurements, an additional benefit is it can penetrate vegetation more easily than passive sensors (in the gully the scanner was able to penetrate the spinifex and foliage to capture the ground and surrounding topography). However, since the measurements are discrete, the sampling resolution means that the point cloud is sparser than other methods. The point resolution is restricted to the size of the laser beam spot size and cannot be increased by simply decreasing the distance to the object.

This specialised equipment requires a trained operator or experienced spatial professional to achieve task deliverables and manage quality assurance. Access limitations can restrict the use of TLS. On this survey, scanning was restricted to the lighter TX5 on one occasion, as transporting the bulkier $\mathrm{C} 10$ on foot up the $800 \mathrm{~m}$ rocky gully proved too difficult. While the open source software can be used, in most cases specialised software is required to extract, register and process the data.

With positional certainty, repeat measurements or reconstruction can be achieved, but the quality of the visualisation experience may be of a lower quality than photogrammetric approaches.

\section{Photogrammetry}

Photogrammetry is a powerful tool for rock art recording at close range. Proximity to the object allows for high precision identification of all features and enables high resolution 3D point clouds to be created. Sub-millimetre accuracy is also possible under ideal conditions and through the use of a high quality sensor. Furthermore, additional spatial information and relationships can be extracted based on the images which can then be used to undertake further archaeological analysis of the rock art motifs. The time required to collect the images in the field is short (30-60 $\mathrm{min})$. And the equipment is easier to carry in difficult terrain.

Compared to laser scanners, cameras normally capture wavelength within the visible light, i.e. mostly what a human eye can see. Some variations from this are possible and depend on the camera sensors' sensitivity. Cameras provide the opportunity to capture a more realistic and three dimensional view of the object. These images can also be easily further manipulated with programs such as D-Stretch to enhance the visibility of less visible and hard to spot rock art. In addition, the combination of such images with Laser Scanning information offers a great opportunity when analysed together as one method overcomes the other methods drawback [32, 33].

However, because photogrammetry is a passive sensor system, it requires an external light source to illuminate the scene; a major disadvantage is sensitivity to changing light conditions and a dependency on nadir or near nadir vantage points for field capture. Insufficient light means it will not be possible to capture meaningful images. While most cameras can adapt to the light conditions, they only can adapt to either the bright or the dark areas. So, while part of the rock art may be illuminated by the sun, it is not possible to capture the rock art located in a shaded area. It is possible to increase the light sensitivity by changing the ISO setting of the camera: a high ISO value means it is possible to capture images with information even at night times without having to use a light source. However, a higher ISO value also introduces noise into the images leading to a decrease of the precision of the derived 3D information.

Any vegetation in the scene will obscure the photographic exercise, unlike laser scanners which 'see through' a background covered by the vegetation. Vegetation often produces noisy 3D point clouds requiring a time-consuming post data collection manual clean upas in most instances the vegetation cannot be simply removed.

\section{Photographic reconstruction}

Photographic reconstruction enables key archaeological features to be visualised at very high 3D resolution and enables further analysis and interpretation. This is possible as compared to the previous method a key focus is on rendering to create a visual realistic and appealing picture of the reality. 
The data capturing is fast, and the costs of the hardware (based upon standard cameras and lenses) are relatively low and limited training is required. The size and weight of the equipment also made it more logistically practical to take to the more remote sites that were recorded.

Photographic reconstruction is far less prone to missing geometry than laser scanning as each photograph is intentionally taken from a different position, and the positions chosen to maximise coverage and minimise concave areas. The photographs can deliberately target key features and can capture inaccessible areas such as crevices, holes and around and between rocks. In addition, the resolution of the reconstruction can be varied, for example areas of higher importance can be photographed more carefully and densely resulting in a more detailed mesh.

It is often claimed that an advantage of photographic $3 \mathrm{D}$ reconstruction is that it does not need a skilled operator, but it is generally the case that the quality of the resulting model are highly dependent on the photographic techniques.

The collected photo data is amenable to visual outputs such as virtual reality, real-time navigation and interpretive walkthroughs. Point cloud data is not well supported in mainstream virtual reality or gaming platforms and point clouds data suffers from a number of issues particularly with the loss of data resolution when zooming in on objects. The high quality textures of the photographs are also more visual representative of the surface of the object than those produced by a point cloud. Textured meshes, for example can be imported into all of the mainstream 3D modelling, editing and animation packages.

The same drawbacks which exist for the photogrammetry solution also are relevant for the photographic reconstruction, i.e. namely the sensitivity against light. Another major drawback is that while the data look visual attractive it is not suitable to take 3D measurements due to the lack of metric information. This means that in contrast to laser scanning and photogrammetry spatial analysis starting with basic measurements of distances to further analysis such as the roughness of surfaces is not possible. In addition, while the point cloud based on photographic reconstruction may not show so many holes such as point clouds from laser scanners or photogrammetry, it does not mean that they are not there. They are more difficult to spot in the rendering because the human eye is able to interpolate where there maybe issues present.

\section{Deliverables/products}

\section{Measurement/recording}

One of the most important considerations of these approaches involves their ability to capture the desired data. This is closely followed by the need to interrogate and extract information and measurements from the data once you leave the field. This is vital given that the time on site is often limited, that many heritage sites are remote and that repeated visits are not always possible. Examples of the 3D data are provided in Fig. 5.

For simple measurements such as size and position of the rock art, all three techniques are equally reliable. Laser scanning offers a slightly better advantage for longer range measurements (i.e. distance between panels, the locations of panels relative to the rock shelter and to the overall site topography). While it can also be used to record the shape and size of the rock art, photogrammetry provides a better option for providing fine scale resolution. The resolution for the geometric information from photogrammetry can be increased by simply decreasing the distance to the object of interest. An example is given of engravings and the characterisation of their depth (see Fig. 6).

An important aspect of rock art recording is information on the pigmentation and texture of the art. In this case photographic imagery is best suited as it can be calibrated to correct for errors in the radiomimetic capture, and is automatically applied to the geometric information of the points and surface. The laser scanner lacks this resolution and relies on intensity data. While this allows for differentiation of the rock art to the background based on spectral reflectance strength, it does not allow for the adequate pictorial documentation and capturing (Fig. 7).

Photogrammetry and laser scanning can be used to map discrete rock art features. While identification in the laser scanning point cloud can be challenging, the visibility of the rock art in the images is easier. Rock faces can be quite irregular, so the identifying corresponding discrete points in the image can be difficult (see Fig. 8). The digitised rock art can be imported into CAD software or any other site management software for this type of measurement.

\section{Topographic mapping}

Another important aspect of the data capture is the ability to map the surrounding locations and features. Not only does this allow plans and drawings to be generated of the site, but also the pigment art and engravings to be put into their landscape context. It can also show the effects of the local environment on the art: such as whether it is exposed to sunlight, whether there is a danger of erosion from water runoff or from exposure to the elements.

A laser scanning point cloud captures the entire site, enabling virtual survey and mapping of all site features and topology. The location of the art and the extents of the site and its surrounds can also be measured and digitised (see Fig. 9: where the planar view and a rock shelter cross-section have been digitised). 


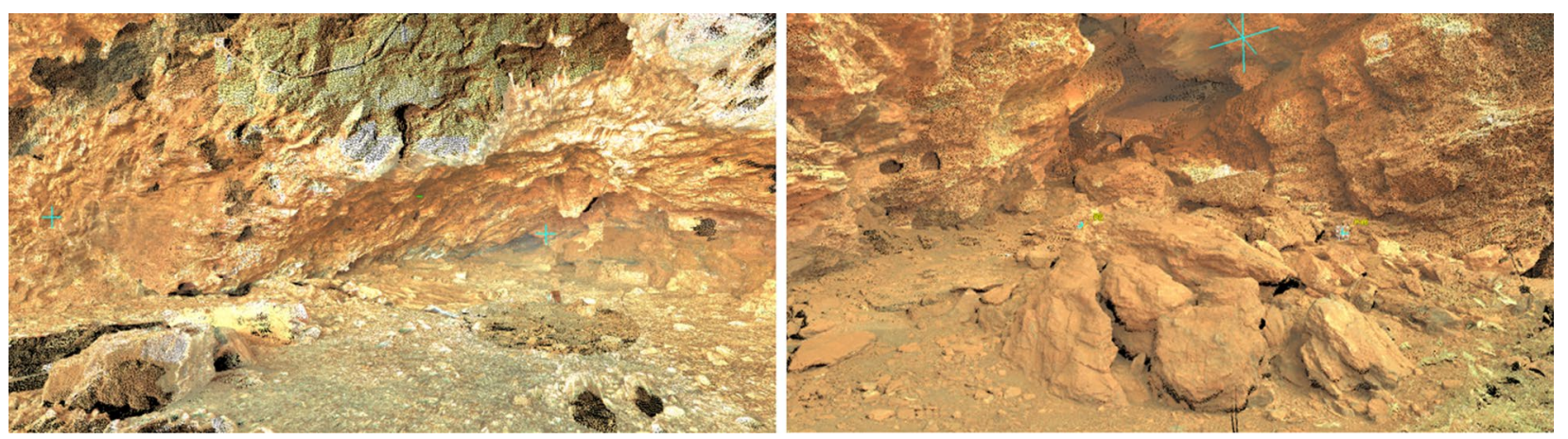

Fig. 5 Screenshot examples of 3D point cloud (here: DE-SH2)

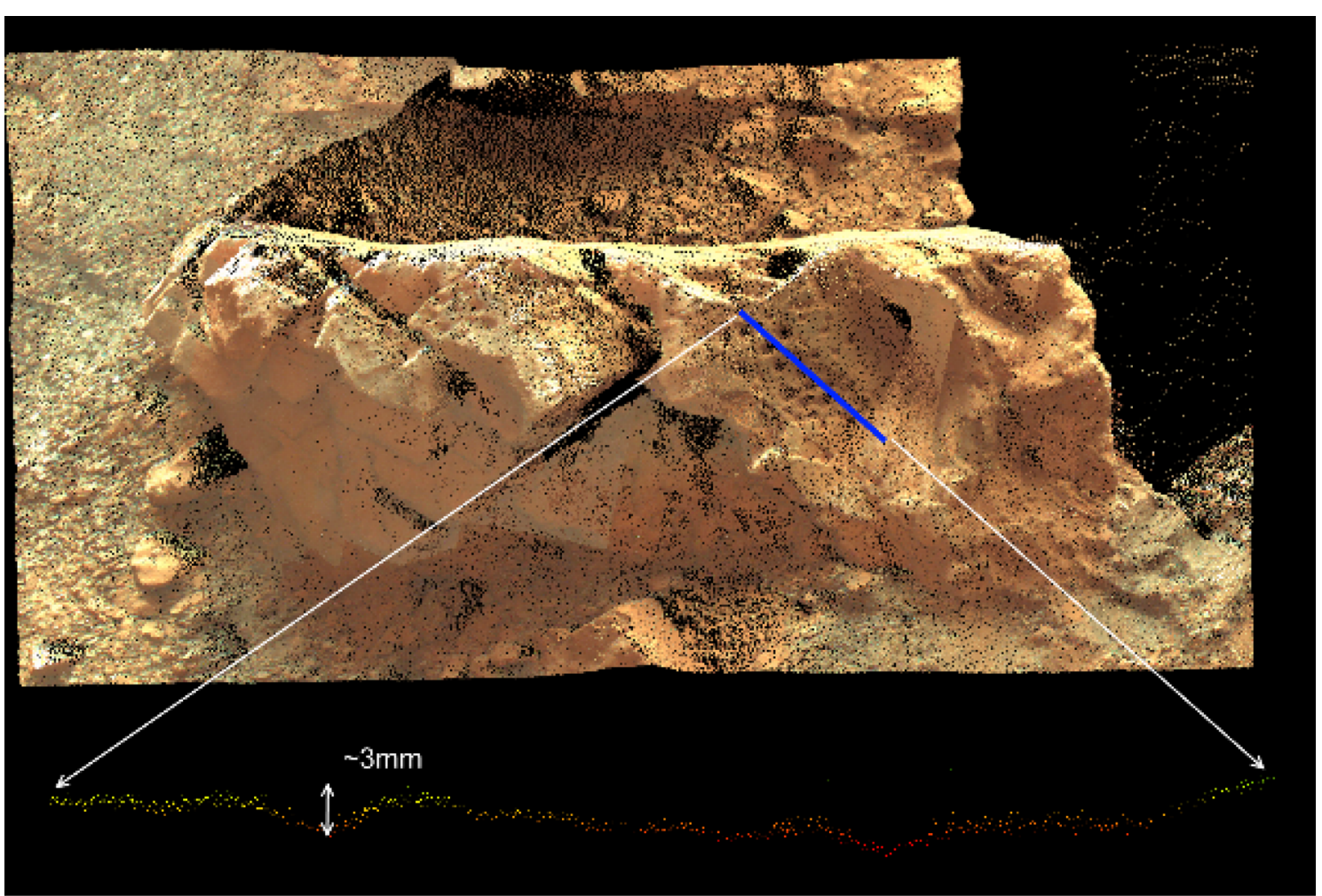

Fig. 6 Cross section from pecked pits found at $\mathrm{DE}-\mathrm{SH} 2$

When mapping the elevation contours and some of the features, dense vegetation can cause issues with extracting the ground and terrain surface (see Fig. 10 where the grass extends approximately $1 \mathrm{~m}$ about the ground). Because the laser scanner can penetrate such vegetation, all the points above the lowest points in region can be removed (Fig. 11), leaving only the ground points to model the terrain. It is more difficult to remove vegetation from the photogrammetry data than from the laser scanning point clouds. The removal of vegetation allows a better analysis of the rock art location in its local setting. Based on the terrain information it is possible to assess if sites are in danger during heavy weather events, e.g. flooding, because water run-off can be assessed.

\section{D reconstruction}

$3 \mathrm{D}$ reconstruction is not limited to photographic reconstruction but can also be achieved using point clouds derived from laser scanners and photogrammetry, as well as other potential sensors such as ultrasonic and radar. 


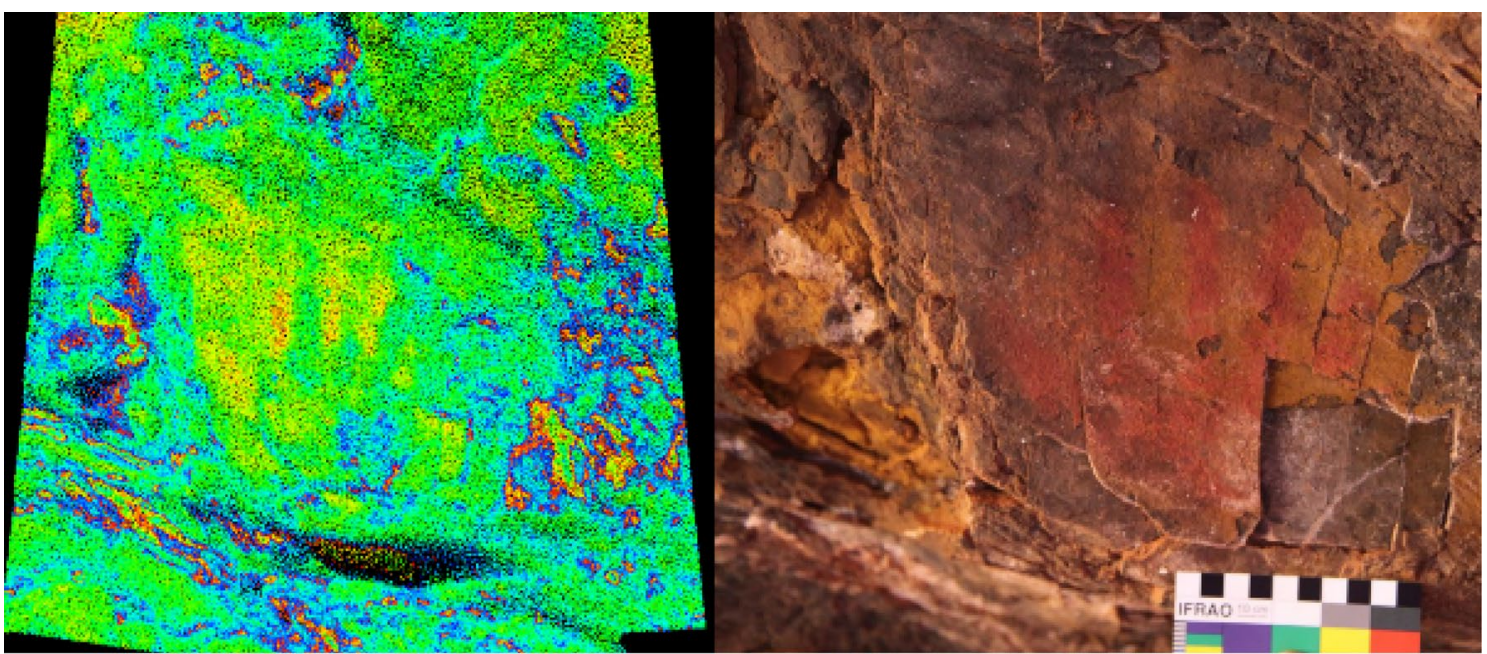

Fig. 7 Coloured intensity values captured by the TLS system (left) and the corresponding RGB image (right)
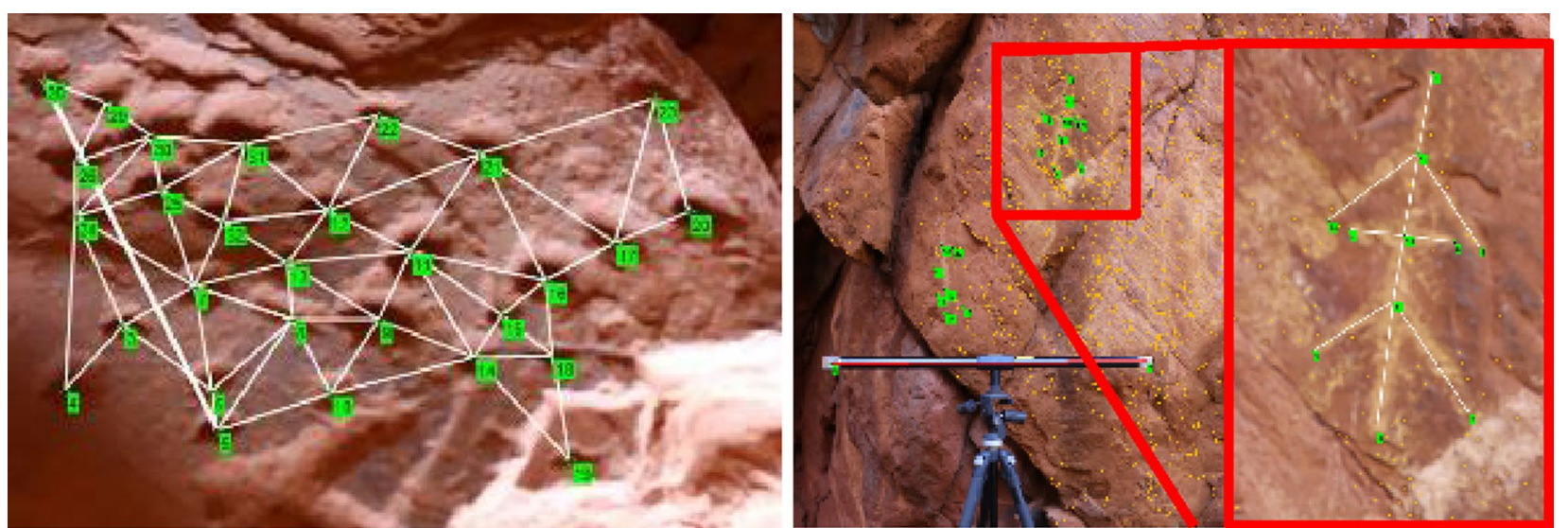

Fig. 8 Digitised rock art

While photographic reconstruction is a fast photographic based recording technique resulting in highly versatile textured meshes, photogrammetry and laser scanning point clouds and mesh offer the opportunity of 3D measurements but are constrained by their lack of visual appeal. Because of the visual appeal of photographic reconstruction the resulting meshes are well-suited for integration into animation, visualisation and virtual reality applications. Being photography based there is scope for dealing with variable lighting conditions. Multiple exposure high dynamic range (HDR) photography can be deployed using a tripod, although a consequence of this is longer recordings times.

With laser scanning, while the metric and geometry of the captured objects can be derived and therefore are known, and an accuracy measure for any point in the point cloud can be derived, the texturing and visualisation will be poor due to the quality of the onboard imaging sensor. This could be improved by mapping the images onto the point cloud and mesh. However, the point cloud from photogrammetry or photographic reconstruction already has good texture and visualisation properties. The disadvantage is that without good control to constrain the extracted surface, it may deform and warp away from the true surface representation. The scanner is more robust against this issue, and can be used to constrain the surface from the imagery (Fig. 12).

\section{Representation/communication}

As is often the case with most technologies communicating the end product of the laser scanning and photogrammetry to various stakeholders was challenging when limited time and budget was available for postprocessing and reconstruction. The expectation from all 


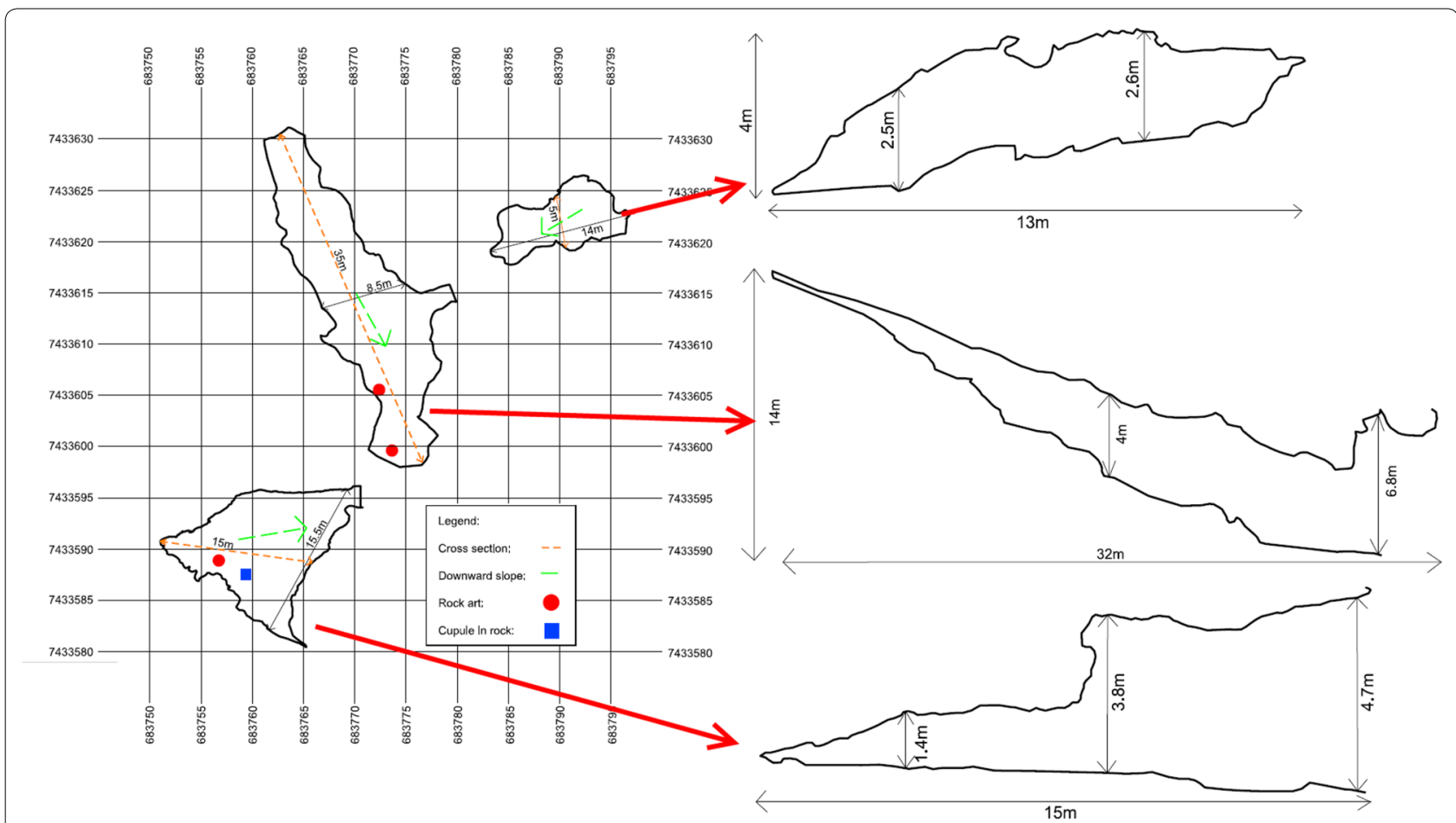

Fig. 9 2D drawings of site DE-SH2 including a planar view of the rock shelters (left) and the cross sections (right) extracted from digital point cloud data

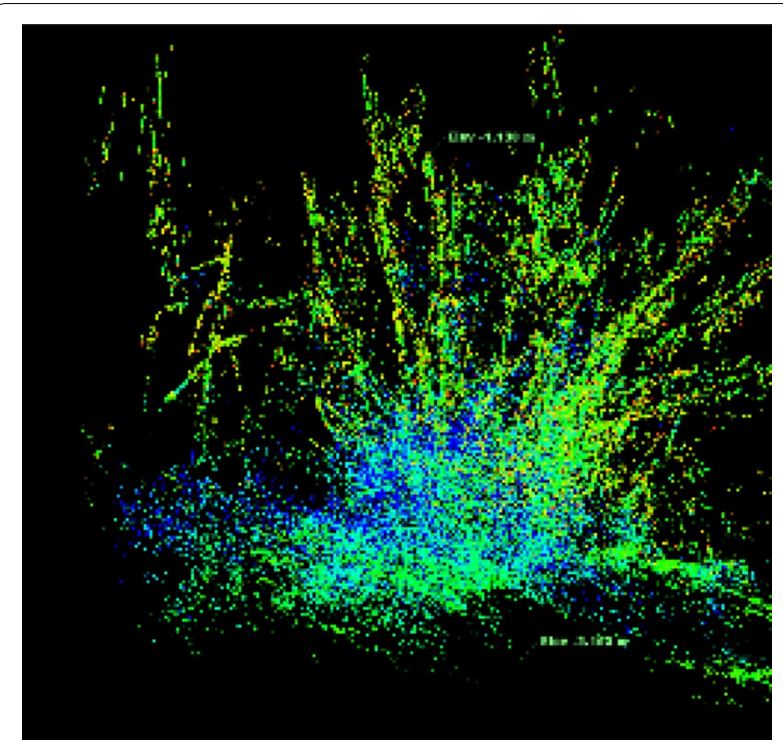

Fig. 10 Long grass captured by scanner

stakeholders was that the project would produce a readily usable output for education and interpretation. While this is possible, delivering a polished end-product takes many weeks of work. This project required the input of spatial science experts and on site surveyors to produce a result that could be used for reconstruction. Without the support of these experts this project would have been difficult to deliver. The output produced from the laser scanning is not readily accessible by non-spatial experts without specialist software and required the use of video flythrough and screen shots to enable the data to be visualised by the Traditional Owners. For this project video flythroughs were created showing the laser scan data however for the community this type of output was much harder to connect to the real landscape on the ground. Video of the reconstructed cave is provided in the Additional file 1 to this paper.

The true shape of the pecked pits and an engraved bird track found in one shelter is best visualised through a 3D output, especially compared to traditional rock art recording methods. For this project the $3 \mathrm{D}$ models were integrated into unity to build a virtual walkthrough of the rockshelters and their archaeological features. This type of output enabled members of Yinhawangka that were unable to visit the site to virtually view the key archaeological features in detail at a meeting in Paraburdoo. This assisted the Traditional Owners in making decisions in relation to the ongoing management of the sites (Fig. 13). The use of 3D visualisation and virtual reality during meetings about the sites' management provided a 

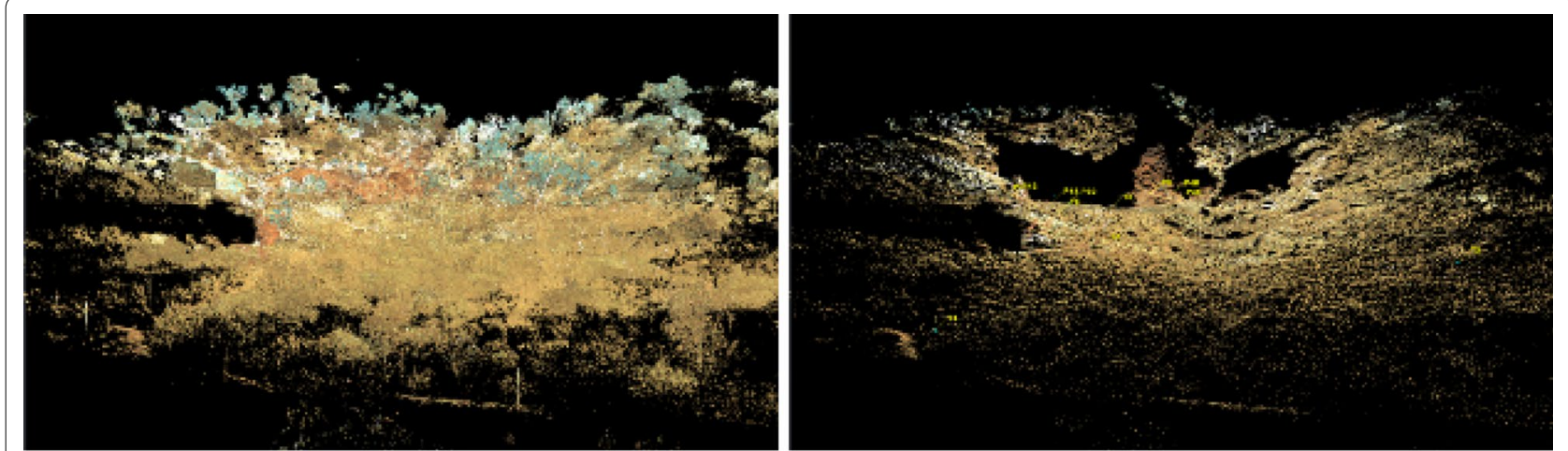

Fig. 11 Point cloud with vegetation (left) and without vegetation (right) in the gully of site DE-SH1

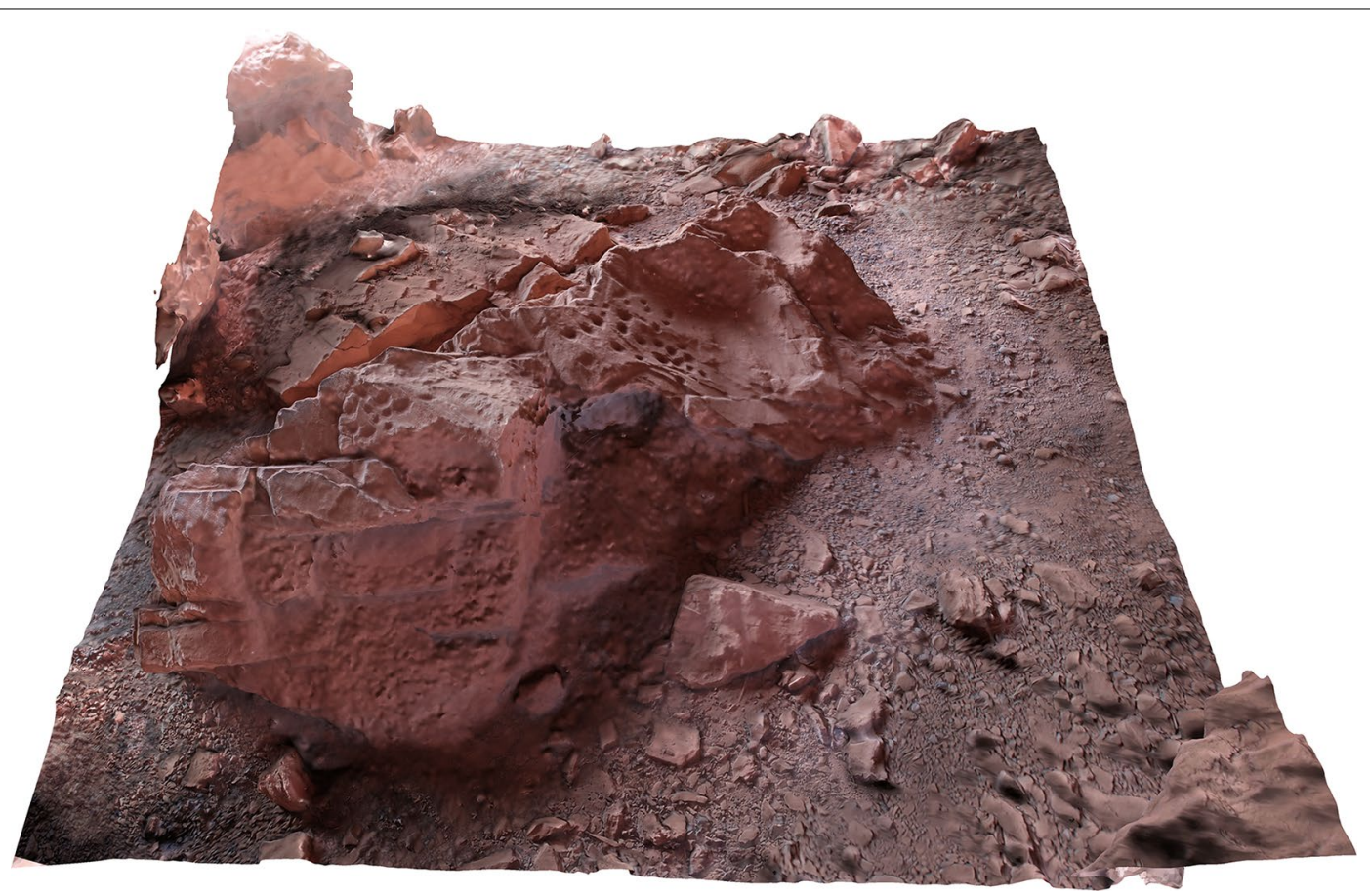

Fig. 12 Textured 3D surface reconstruction

realistic recreation of the site and enabled key community decision makers to make informed decisions about their management.

\section{Discussion}

For this project the pairing of high resolution scanning, with photogrammetry, photographic reconstruction and rock art recording enabled a detailed and comprehensive picture of the rock art of West Angelas to be created. The visual outputs generated through these techniques coupled with additional ethnographic surveys have assisted with interpretation and management of these sites. All of the methods outlined in these methods have enabled
Rio Tinto to pro-actively engage with Yinhawangka Traditional Owners in relation to the ongoing management of the sites.

The laser scanning and photogrammetry data collected prior to the commencement of mining has established a spatially reliable baseline record for sites that are located in close proximity to mining operations and that are at potential risk of indirect impacts from blasting. This baseline data can be used in conjunction with other existing geotechnical monitoring programs to measure changes over time when compared to data collected from additional scans over different time periods. For the Yinhawangka 


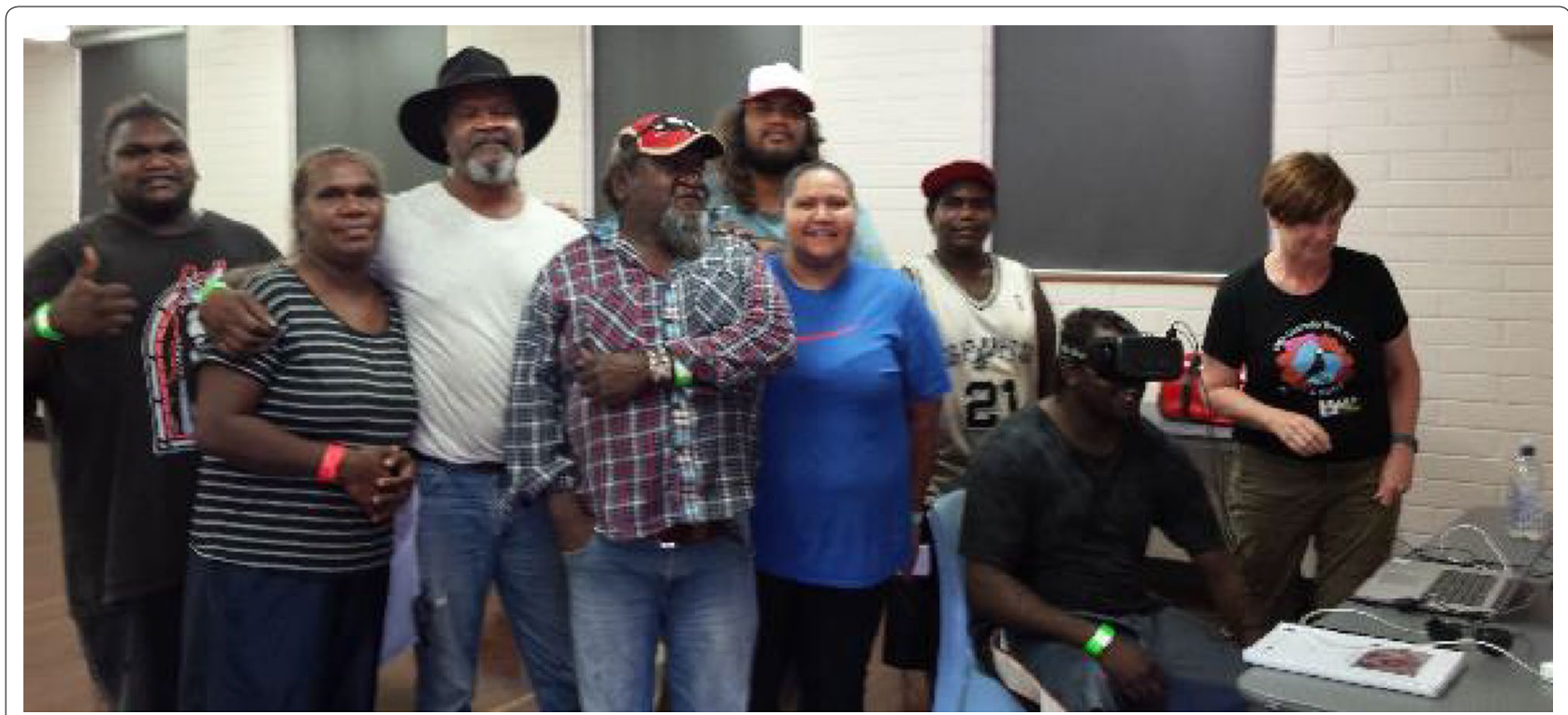

Fig. 13 Virtual reality site visit being viewed by Yinhawangka Traditional Owners

Traditional Owners however laser scanning data does not provide a real sense of place due to the lack of imagery.

The combination of photographic reconstruction, rock art and ethnographic data has provided the Yinhawangka people with significant opportunities for better understanding these sites and their regional context. The high textured outputs that 3D reconstruction and photogrammetry can provide were embraced by the Yinhawangka Traditional Owners and a sense of being 'virtually' there was created. These outputs can be used to facilitate further recording, management, research and interpretation of rock art in Yinhawangka country. The proximity of one of the case study sites to an existing mine pit means that regular access by the Aboriginal community is not possible due to safety issues. The high resolution baseline data can be used to create a complete digital and physical (e.g. $3 \mathrm{D}$ print) reconstruction of the cave and its archaeological features for the Aboriginal community.

\section{Conclusion}

Like traditional surveying, there is often a suite of tools and technologies required for a desired outcome and output. The operator must assess the most effective tool to meet the desires of the customer and end user requirements.

Digital image and laser based technologies are available to archaeologists and Traditional Owners to assist in the analysis, interpretation, visualisation and management of heritage sites. In a cultural heritage management context, the combination of laser scanning and photogrammetry reconstruction can assist in the generation of spatial and visual records of heritage sites and their archaeological features. Spatial and visual technologies such as laser scanning and photogrammetry can assist researchers with accurate spatial and contextual data, Traditional Owners with visual and interpretive outcomes for their heritage using photographic reconstruction, and industry in the proactive ongoing management of site conditions within project areas. For highly significant heritagesuch as rock art sites-these technologies represent significant opportunities for understanding, interpreting and managing the rock art as well as providing unique educational and community interpretive opportunities.

The technologies applied in this project may not be appropriate in all contexts. It is important to select the right technology for the job at hand. Interdisciplinary collaborations between archaeologists, technical specialists and heritage managers and the overarching interest from Traditional Owners will always produce the best outcomes.

\section{Additional file}

Additional file 1. Flythrough of 3D cave reconstruction-DF-SH1.

\section{Authors' contributions}

This RTIO community and research project was managed by AD, who also facilitated the engagement and consultation with Yinhawangka Traditional Owners. JM and Meg Berry recorded the rock art which was analysed and reported on by JM. DB and PH collected and analysed the laser scanning data. PH undertook the photogrammetry analysis. PB undertook the high resolution photography, 3D reconstruction and visualisation. JM and AD presented the visualisation outputs to the Yinhawangka Traditional Owners at their annual 
general meeting in Paraburdoo in 2015. All authors read and approved the final manuscript.

\section{Author details}

${ }^{1}$ Rio Tinto, Communities Division, PO Box A42, Perth, WA 6837, Australia.

${ }^{2}$ Department of Spatial Sciences, Curtin University, Perth, WA, Australia. ${ }^{3}$ The University of Western Australia, Perth, WA, Australia. ${ }^{4}$ Centre for Rock Art Research and Management, The University of Western Australia, Perth, WA, Australia.

\section{Acknowledgements}

This research project was completed under contract between CRAR+M UWA and Rio Tinto. The field team consisted of archaeologists Jo McDonald and Meg Berry (CRAR+M UWA), visualisation specialist Paul Bourke (then at iVEC@UWA) and surveyors David Belton and Petra Helmholz (Spatial Sciences Department at Curtin University). The team was accompanied by five Yinhawangka Traditional Owners: David Cox ("Barndu"), Clayton Parker, James Cox, Churchill Jones and Eric Galby Snr; and Rio Tinto representatives Bob Tait and Annabelle Davis. This project would not have been possible without the on-ground support and technical advice of the Rio Tinto survey team and Yamatji Marlpa Aboriginal Corporation who assisted with the co-ordination of Traditional Owner representatives for the survey. The team also gratefully acknowledge Yinhawangka Aboriginal Corporation in their support for this paper.

\section{Competing interests}

The authors declare that they have no competing interests.

\section{Funding}

The project referenced in this paper was fully funded by Rio Tinto in the context of a cultural heritage management study at Rio Tinto's West Angelas operations.

\section{Publisher's Note}

Springer Nature remains neutral with regard to jurisdictional claims in published maps and institutional affiliations.

Received: 30 November 2016 Accepted: 10 May 2017

Published online: 04 July 2017

\section{References}

1. Scopigno R, Callieri M, Cignoni P, Corsinin M, Dellepiane M, Ponchio F, Ranzuglia G. 3D Models for cultural heritage: beyond plain visualization. IEEE Comput Soc. 2011;2011:48-55.

2. Dellepiane M, Dell'Untro N, Callieri M, Lindgren S, Scopigno R. Archaeological excavation monitoring using dense stereo matching techniques. J Cult Herit. 2013;2013(14):201-10.

3. De Reu J, De Smedt P, Herremans D, Van Meirvene M, Laloo P, De Clercq W. On introducing an image-based 3D reconstruction method in archaeological excavation practise. J Archaeol Sci. 2014;41:251-62.

4. Koutsoudis A, Vidmar B, loannakis G, Arnaoutoglou F, Pavlidis G, Chamazas C. Multi-image 3D reconstruction data evaluation. J Cult Herit. 2014;15:73-9.

5. Westoby MJ, Brasington J, Glasser NF, Hambrey MJ, Reynods JM. 'Structure-from-Motion' photogrammetry: a low-cost, effective tool for geoscience applications. Geomorphology. 2012;178:300-14.

6. Georgopoulos A, loannidis C. Photogrammetric and surveying methods for the geometric recording of archaeological monuments. FIG Working Week. 2004

7. Dell'Uto N, Leander AM, Dellepiane M, Callieri M, Ferdani D, Lindgren S. Digital reconstruction and visualization in archaeology — case-study drawn from the work of the Swedish Pompeii Project. In: Addison A, Guidi G, De Luca L, Pescarin S, editors. Digital Heritage International Congress; 2013: p. 621-28.

8. Arbace L, Sonnino E, Callieri M, Dellepiane M, Fabbri D, Idelson Al, Scopigno R. Innovative uses of 3D digital technologies to assist the restoration of a fragment terracotta statue. J Cult Herit. 2013;14:332-45.
9. Siotto E, Dellepiane M, Callieri M, Scopigno R, Gratziu C, Moscato A, Burgio L, Legnaioldi S, Lorenzetti G, Palleschi VA. Multidisciplinary approach for the study and the virtual reconstruction of ancient polychromy of Roman sarcophagi. J Cult Herit. 2014;16(3):307-14.

10. Vosselman G, Mass H-G. Airborne and terrestrial laser scanning. Boca Raton: CRC Press; 2010.

11. Mudge M, Schroer C, Noble T, Matthews N, Rusinkiewicz S, Toler-Franklin C. Robust and scientifically reliable rock art documentation from digital photographs. A companion to rock art. In: McDonald J, Veth P, editors. Malden: Taylor and Francis Publishing; 2010. p. 644-59.

12. Cultural heritage imaging. http://culturalheritageimaging.org/Technologies/Photogrammetry/index.html. Accessed 17 Nov 2016.

13. McDonald J. Detailed recording, photogrammetry and laser scanning of four rock art sites at West Angelas, East Pilbara. 2015, CRAR+M UWA (unpublished report to RTIO).

14. Misra P, Enge P. Global positioning system: signals, measurements, and performance. 2nd ed. Lincoln: Ganga-Jamuna Press; 2010.

15. Smith S, McDonald J, Balme J, MacLaren G, Paterson A. Creating a paperless recording system for Pilbara rock art. Across space and time. In: Traviglia A, editor. Papers from the 41st conference on computer applications and quantitative methods in archaeology. Chicago: University of Chicago Press. Perth, 25-28 March 2013; 2015. p. 89-96.

16. D-Stretch. http://www.dstretch.com/. Accessed 17 Nov 2016.

17. Stevens R. Preliminary advice following an Yinhawangka ethnographic site visit at rockshelter site DE-SH2 and an ethnographic site assessment of DF-SH1 at West Angelas. 2014, Stevens Heritage Services (unpublished report to Rio Tinto)

18. Leica geosystems, Leica ScanStation C10 datasheet. http://www.hds. leica-geosystems.com/en/Leica-scanStation-c10_79411.html. Accessed Nov 2016.

19. Trimble TX5 laser scanner datasheet. http://www.mep.trimble/literature/ datasheet/trimble-tx5-laser-scanner-datasheet. Accessed Nov 2016.

20. Besl PJ, McKay ND. A method for registration of 3-D shapes. IEEE Trans Pattern Anal Mach Intell. 1992;14(Suppl 2):239-56.

21. iWitness close range photogrammetry, iWitness Pro V3.http://www. iwitnessphoto.com/products/iwitnesspro.html. Accessed 17 Feb 2014.

22. Rothermel M, Wenzel K, Fritsch D, Haala N. SURE: photogrammetric surface reconstruction from imagery.In: Proceedings LC3D workshop, Berlin, Dec 2012.

23. Agisoft photoscan professional edition. http://www.agisoft.ru/products/ photoscan/professional/Accessed. Accessed 17 Feb 2014.

24. Wu C. VisualSFM: a visual structure from motion system. http://homes. cs.washington.edu/ ccwu/vsfm/. Accessed 13 Feb 2014.

25. Lowe DG. Object recognition from local scale-invariant features. In: proceedings of the international conference on computer vision. 1999; vol. 2, p. 1150-57.

26. Hirschuller $\mathrm{H}$. Stereo processing by semi-gobal matching and mutual information. IEEE Trans Pattern Anal Mach Intell. 2008;30(Suppl 2):328-41.

27. Furukawa Y, Ponce J. Accurate, dense, and robust multi-view stereopsis. IEEE Trans Pattern Anal Mach Intell. 2010;32(Suppl 8):1362-76.

28. Semyonov D. Algorithms used in photoscan. http://www.agisoft.ru/ forum/index.php?topic=89.0. Accessed 13 Feb 2014.

29. Snavely N, Seitz SM, Szeliski R. Modelling the world from internet photo collections. Int J Comput Vis. 2007;80(2):189-210.

30. Bakula K. Multispectral airborne laser scanning - a trend in the development of LiDAR technology. Archiwum Fotogrametrii Kartografii i Teledetekcji. 2015;27:25-44.

31. Bakula K, Kupidura P, Jelowicki L. Testing of land cover classification from multispectral airborne laser scanning data. In: The international archives of the photogrammetry, remote sensing and spatial information sciences; 2016; vol. XLI-B7, p. 161-9.

32. Skoog B, Helmholz P, Belton D. 2016. Multispectral analysis of indigenous rock art using terrestrial laser scanning. The international archives of the photogrammetry, remote sensing and spatial information sciences; 2016; vol. XLI-B5, p. 405-12.

33. Buchon-Moragues F, Bravo JM, Ferri M, Redondo J, Sanchez-Perez JV. Application of structured light system techniques for authentication of wooden panel painting. Sensors. 2016;16(6):881. doi:10.3390/s16060881. 